\title{
Mechanical Circulatory Support as Bridge to Pediatric Heart Transplantation
}

\author{
Martin Schweiger and Michael Huebler \\ Additional information is available at the end of the chapter \\ http://dx.doi.org/10.5772/intechopen.76144
}

\begin{abstract}
Fueled by the uncertainty and the time required to obtain a donor heart, mechanical circulatory support (MCS) forms an essential part of end-stage heart failure. Extracorporeal membrane oxygenation (ECMO) use is limited to a few days before serious complications like bleeding occur. Prolonged support in terms of ventricular assist device (VAD) as a bridge to transplantation (BTT) became mandatory to overcome death on the waiting list. Within the last decade, VADs in adults have evolved drastically with the introduction of continuous flow (cf) devices. Increased miniaturization of VADs and new support strategies have increased its use in the pediatric population even in small children and patients with congenital heart disease (CHD). Nevertheless, patient and device selection in this patient population remain challenging to achieve optimal outcome and decrease complication rates. This comes with the need for care providers specialized in this field. Size issues and anatomical diversity make decision making complex and unique when compared to general adult practice. Neonates with single ventricle physiology are the highest risk candidates for VADs. This chapter reviews the most relevant durable VADs used in children including the rapid evolution of using adult designed cf-VADs to support children with anatomical normal hearts and CHD.
\end{abstract}

Keywords: pediatric ventricular assist device, pediatric heart transplantation, bridge to transplantation, congenital heart disease, Berlin Heart EXCOR pediatric

\section{Introduction}

Hospitalization among children suffering from end-stage heart failure (HF) is increasing [1]. If not otherwise correctable and in the absent of contraindications, heart transplantation 
(HTx) remains the treatment of choice. Pediatric HTx (pHTx) represents a small but very special part in the field of cardiac transplantation. Children remain at an increased risk of death on the waiting list for HTx [2]; especially infant heart transplant recipients are at a greater risk of death compared to older children. The main reason is the search for an appropriately sized organ donor [2-4]. The limited numbers of available pediatric donor heart organs led to an increased mean waiting time in most Western countries [4]. Tapping all potential brain-dead donors and expanding the recipient pool on an international level is thus of vital importance especially for smaller countries in Europe. Therefore, international organ exchange among organ procurement organizations seems to be essential and has a direct positive impact on the chances of patients to get a timely, often life-saving transplantation [5]. All these efforts have, however, not resulted in a decreasing waiting time on the waiting list.

Fueled by the uncertainty and the time required to get a donor heart, mechanical circulatory support (MCS) as a bridge to transplantation (BTT) became mandatory to overcome death on the waiting list. Historically, MCS was developed if weaning from cardiopulmonary bypass $(\mathrm{CPB})$ was not possible to allow for a recovery. Therefore, all centers performing congenital heart surgery have experience with extracorporeal membrane oxygenation (ECMO). Its use, however, is timely limited (days to weeks) before serious complications like bleeding occur [6]. Further, ECMO application is limited to short-term support due to immobilization of the patient and the patient must remain on the intensive care unit (ICU). Ventricular assist device (VAD) was shown to be superior to ECMO support, considering the increased risk of 1-year mortality associated with EMCO support $[6,7]$.

While VAD use in children is gaining more attention, there are several challenges to consider. On anatomical and physiological grounds, three different groups can be distinguished: adult patients with anatomic normal heart, pediatric patients with anatomic normal hearts, and patients with congenital heart disease (CHD) irrespective of age. There are clear differences in the pathophysiology of HF compared between adults, children, and CHD patients. Hospitalization of children suffering from HF due to CHD is increasing [1], while reported survival of children on VAD support suffering from CHD is still low [8, 9].

In adults with structural normal hearts, there is a large variety of different VADs which have proven to be safe for long-term support [10] and have developed as a standard treatment option [11]. For pediatrics, only a few VADs are available for patients with a body surface area (BSA) of less than $1.2 \mathrm{~m}^{2}$ or weight less than $20 \mathrm{~kg}$ [12]. Furthermore, limited data are available as children are excluded in major VAD trials. Only one prospective trail is reported by Fraser et al. using the Berlin Heart EXCOR®. Currently, there are only two VADs designed for children with a body surface area below $1.2 \mathrm{~m}^{2}$ : the Medos HIS and the Berlin Heart EXCOR. Finally, in adult patients, the numbers of BiVAD implantations are declining [13, 14]; the incidence of biventricular failure among children remains high, with over $15 \%$ requiring BiVAD or total artificial heart support [15] and results seem to be inferior to LVAD only [16].

Finally, if a contraindication for HTx like pulmonary hypertension or malignancy is diagnosed, a concept known as bridge to transplantability may be considered. 
All these considerations come with the need for care providers specialized in this field to determine optimal patient and device selection and to improve outcomes and decrease complication rates for new innovative strategies. This chapter focuses on durable VADs as BTT or candidacy in pediatrics.

\section{Durable VAD support in children as BTT}

In the 1970s, modifications of the original "heart-lung machine" like ECMO or extracorporeal centrifugal pumps [17] have been the principal art of cardiac support. With the need for real long-term support, the need for durable VADs became evident. In 1989, Frazier implanted a mechanical assist device in a 9-year-old boy who was successfully bridged to heart transplantation with a Biomedicus (Medtronic, Eden Prairie, MN) centrifugal pump; the supporting time was 12 h. In 1990, the first Berlin Heart EXCOR, in adult size 50-mL pump, was implanted in a 9-year-old child for 1 week with an uneventful postoperative time after heart transplantation [18]. Two years later, in 1992, pumps in sizes of 10, 25, and 30-mL have been devised, and the 10-mL pump was implanted in a 12-month-old child [19]. Two years later, the first Medos VAD (Medos Medizintechnik GmbH, Stolberg, Germany) was implanted successfully as bridge to transplantation [20]. In the last years, there has been an increase in the use of MCS in the pediatric population mainly driven by the development of smaller VADs, namely continuous flow (cf)-VADs.

\subsection{Indication and device selection}

Patient selection and timing remain crucial factors for improving outcomes in VAD recipients. In children with critical peripheral perfusion (i.e., metabolic acidosis; cardiac index of <2.0 1/ $\mathrm{m}^{2} / \mathrm{min}$, mixed venous oxygen saturation of $<40 \%$ ) despite inotropic support, early signs of renal, hepatic, or multiorgan failure without surgical options to correct any residual structural lesions should be considered for MCS. There are only a few contraindications for MCS like malignant neoplastic diseases with a very limited life expectancy, advanced multiorgan failure, complex congenital heart lesions involving intracardiac shunts or irreversible pulmonary failure and severe extracardiac malformations such as chromosomal and genetic syndromes with poor quality of life prognosis [21].

Selection differs significantly within the pediatric group by structural normal hearts or patients with CHD as well as the age and weight/size of the patient $[15,22,23]$. Some VADs are specified for its use in adults or pediatrics; some are licensed according to a specific body surface area (BsA) and/or some for specific weight/size. Contrarily to adults, where intracorporeal left ventricular assist device (LVAD) has become a routine treatment with subsequent discharge home, options for small children are still limited. A large variety of adult-sized ventricular assist devices (VADs) has proven to be safe for long-term support [10] but only a small number of VADs are available for patients with a body surface area (BSA) of less than $1.2 \mathrm{~m}^{2}$ or weight less than $20 \mathrm{~kg}$ [12]. The Medos HIS (no longer on the market) and the Berlin Heart EXCOR are the only two devices currently designed for children with a body 
surface area below $1.2 \mathrm{~m}^{2}$. The development of pediatric-specific cf-VADs (Infant Jarvik) is approved for Investigational Device Exemption by the US Food and Drug Administration on September 30, 2016 [24]. In adult-sized adolescents and some teenagers reaching a BsA of $>1.2 \mathrm{~m}^{2}$, the implantation of a continuous-flow (cf) intracorporeal device LVAD is feasible as results are non-inferior to extracorporeal devices $[15,25,26]$ and discharge from hospital is possible which guaranties a better quality of life [15, 27-30].

\subsection{Berlin Heart pediatric EXCOR}

When speaking about pediatric VAD support, most data are available for Berlin Heart EXCOR (Berlin Heart AG, Berlin, Germany) (see Figure 1). It was specifically designed for small children and is a paracorporeal, pulsatile, pneumatically driven VAD usable

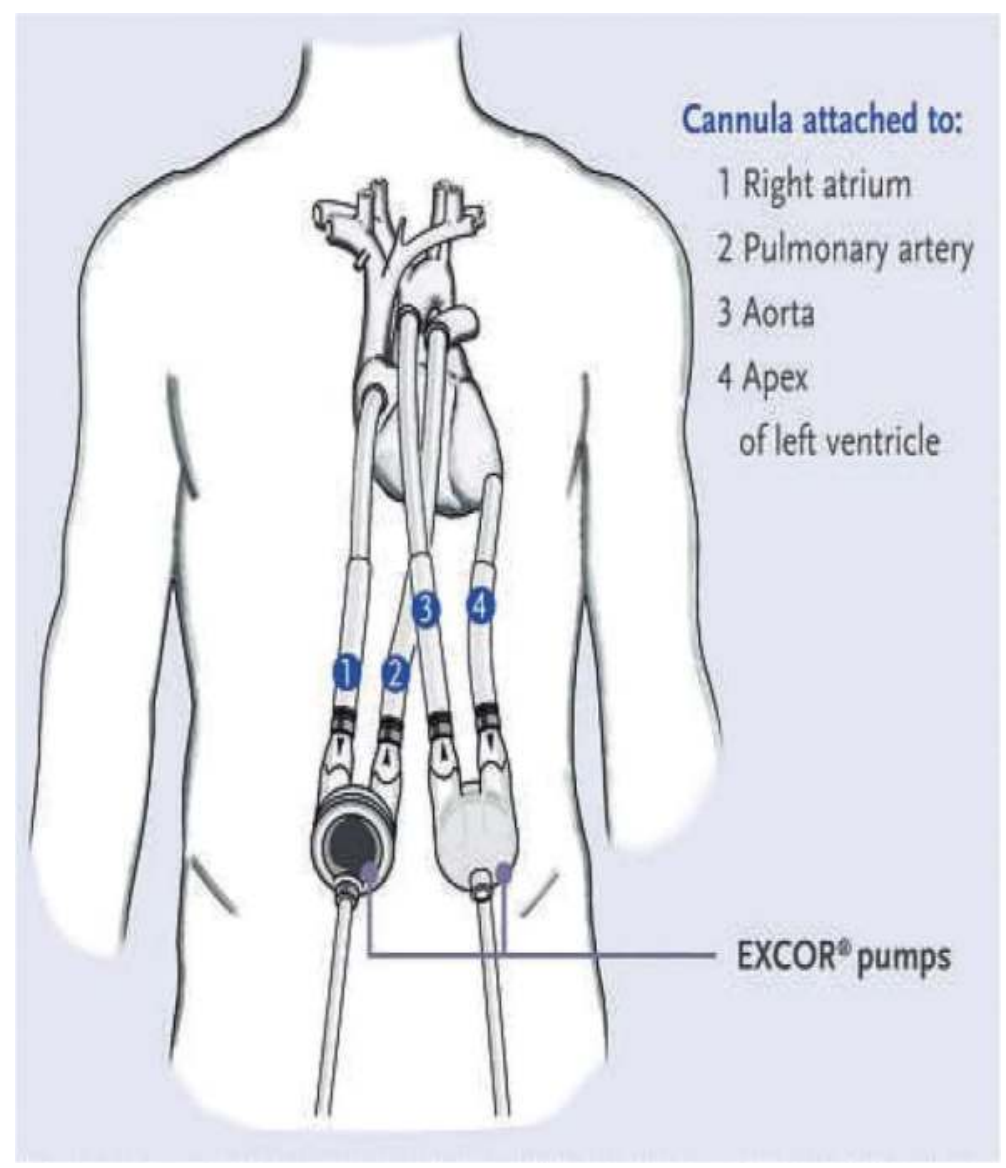

Figure 1. The Berlin heart EXCOR (no permission was asked for reprint). 
for left (LVAD) or biventricular (BiVAD) support. The EXCOR® ventricular assist device (EXCOR) is clinically used since 1990 for the circulatory support of pediatric heart failure in almost 2000 patients as BTT. The blood-contacting surfaces of EXCOR pumps are covalently coated with Heparin (CARMEDA CBAS®, Carmeda, Sweden) to enhance hemocompatibility. The system offers a spectrum of pumps with valves divided into a blood and air chamber and silicone cannula for every body size between $3 \mathrm{~kg}$ and adult size. The pump consists of a translucent, semi-rigid housing of polyurethane. The US investigational device exemption (IDE) multicenter trial examining the safety and efficacy of the device found a better survival for EXCOR compared to ECMO, and serious adverse events, including infection, stroke, and bleeding, were reported with 0.07 events per patient-day in the VAD group and with 0.08 events per patient-day in the ECMO group [6]. The EXCOR was first used in Europe, and the Berlin group gained great experience with the EXCOR even in neonates achieving a survival of 70\% [31]. Nevertheless, the initial North American experience including 73 patients showed that younger age and BiVAD were significant risk factors for death while on the EXCOR [12]. This was confirmed by a recent study concluding that durable VADs should be used very cautiously in children suffering from complex CHD below 1 year of age, especially patients on previous ECMO and those who had prior cardiac surgery [23]. In this study, one-third of all EXCOR patients had CHD, and of these, $30 \%$ had a univentricular physiology [23].

\subsection{Patients with congenital heart disease (CHD)}

Patients, irrespective of age, with CHD represent a unique and difficult patient population to support with VAD/MCS. CHD represents a wide spectrum of cardiac anatomies including the special setting of single ventricle physiologies. Some of the children undergoing CHD surgery are not cured and remain at risk of developing end-stage heart failure. It is estimated that $10-20 \%$ of patients with CHD will require HTx at some point of their life. There is a variety of CHD that results in single ventricle physiology requiring surgical correction ending in the Fontan circulation. HF can occur at any time of the palliative surgery (Norwood stage I, bidirectional cavopulmonary anastomosis, Fontan completion). Large trials investigating the use of MCS in patients with single ventricle are missing. Mainly small series or case reports are published with high mortality rates (i.e., one of three patients surviving to discharge [32-34]) and adverse events, compared to a twoventricular physiology [34]. Support for Glenn circulation has been proven with mixed results [33-36]. Currently available VADs are designed to provide support to the failing ventricle but requirements for VAD systems in the failing Fontan may require cavopulmonary. Nevertheless, available devices have been used for cavopulmonary support in failing Fontan patients [37-42]. For patients with failing Fontan circulation, TAH might be an option [43] (see subsequent text).

By contrast, VAD outcomes in adult CHD patients with two-ventricle physiology are comparable to non-ACHD patients. Most of these patients have a morphologic right ventricle working as systemic ventricle. VAD placement in these patients is possible, and some patients will benefit from VAD support [44-50]. 


\subsection{Biventricular support (BiVAD, TAH)}

The majority of implants in children are only for isolated left ventricular support. However, there is a certain percentage of patients $(\sim 17 \%)$ who require biventricular support with BiVAD or total artificial heart (TAH; see Figure 2) [15]. Results for BiVADS and for TAH (patients $<21$ years) have been reported to be inferior to LVAD only [16, 51]. The Berlin Heart EXCOR remains the "golden standard" for biventricular support in children due to size matters. Case reports and series using two cf-VADs in pediatrics (see Figure 3) with successful BTT with BSA as low as $0.6 \mathrm{~m}^{2}$ have been published [52-55] even in patients with Fontan circulation [56].

\subsection{Anticoagulation and monitoring}

All patients on MCS/VAD support should receive anticoagulation (Class I recommendation) [11]. Thromboembolic events like stroke or pump thromboses in children supported with VAD remain serious adverse events and differ compared to adults [8,57-59]. No standard anticoagulation protocol has been developed so far, and anticoagulation is tailored to different types of VAD and individualized by different centers. To achieve a balance between minimizing thromboembolic events and bleeding complications, an anticoagulation monitoring involving the international normalized ratio (INR), the thrombocyte aggregation test (TAT), and thromboelastography (TEG) has been proposed. The monitoring of unfractionated heparin remains a matter of discussion.

The initial North American EXCOR experience included no consistent anticoagulation protocol [6]. As for the US investigational device trial for the EXCOR, the investigators agreed on the Edmonton protocol. Briefly, this protocol uses a three-drug regimen involving aspirin,

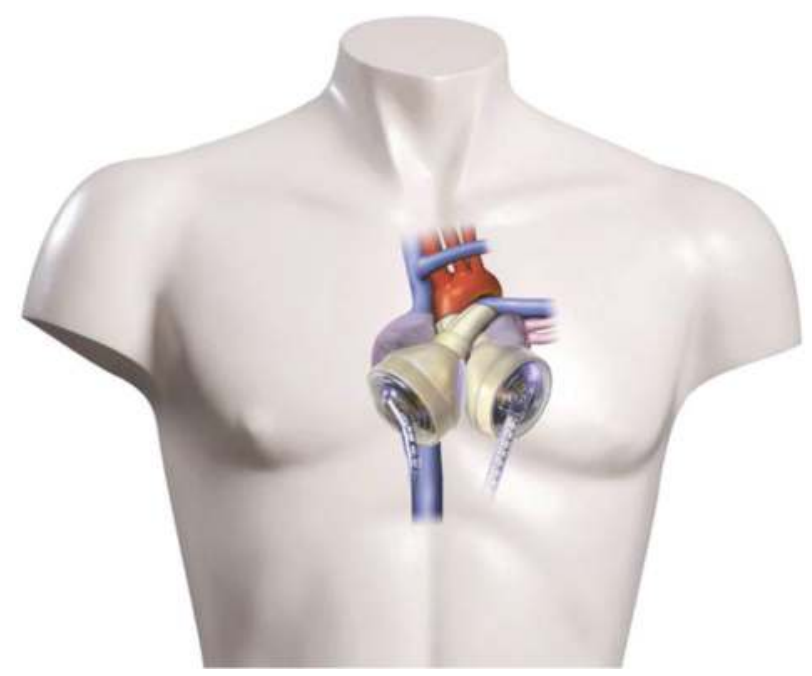

Figure 2. The Cardiowest is a total artificial heart provided by Snycardia (Tucson, AZ, USA). (http://www.syncardia. com/Medical-Professionals/compare-to-bivads.html) (no permission was asked for reprint). 


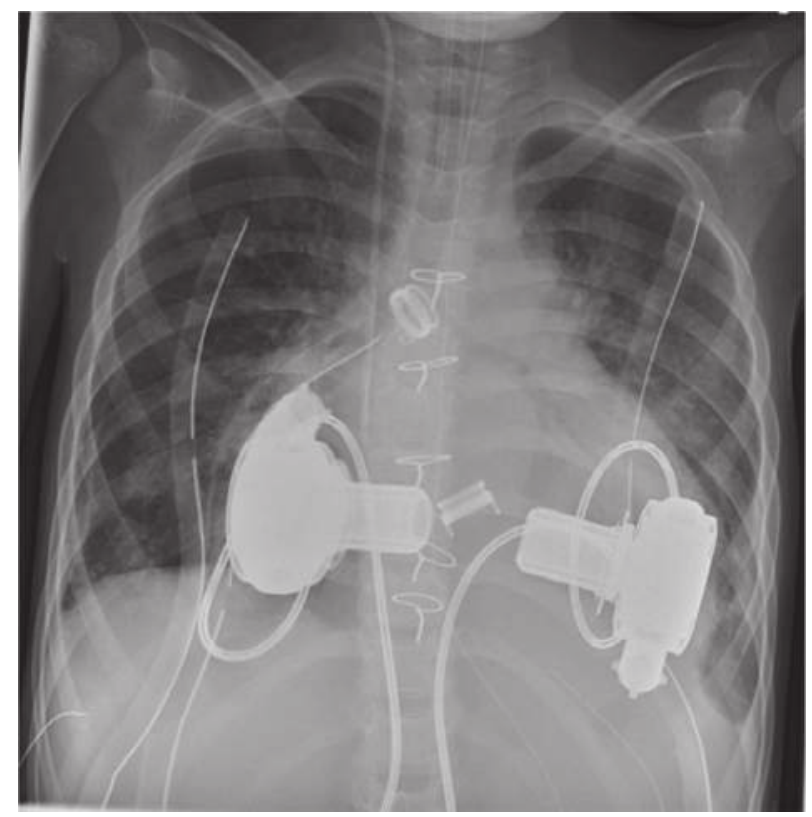

Figure 3. Two intracorporeal VADs for biventricular support in a child with a body weight of $27 \mathrm{~kg}$ (no permission was asked for reprint).

persantine, and enoxaparin or oral anticoagulation [12]. In the immediate postoperative period, unfractionated heparin (UFH) continues to be the anticoagulant of choice, especially in the early postoperative phase in which close titration is required [60]. While the use of UFH is unquestioned, monitoring remains a matter of discussion. Traditionally, in percutaneous coronary intervention or cardiac surgery, the effect of UFH is monitored by the aPTT or the ACT, when higher doses are used in conjunction with extracorporeal bypass. Although aPTT seems to be the standard criterion, it is known that aPTT is susceptible to physiological and nonphysiological factors and may under- or overestimate the level of anticoagulation. For this reason, plasma heparin assays - which determine the anticoagulation activity of UFH by measuring the ability of heparin-bound AT to inhibit FXa-have been proposed. Published data suggest that anti-Xa monitoring achieves therapeutic anticoagulation more rapidly, maintains the values within the goal range for a longer time, and requires fewer adjustments in dosage and repeated tests [61]; further, the aPTT is impacted more frequently by preanalytic compared to anti-Xa [62]. It also may be of particular advantage in pediatric patients (better correlated with heparin dosing than the aPTT or ACT in pediatric ECMO). We at our institution use anti-XA [63], but so far there are too less data available to draw a final solution and both methods are used clinically. After the removal of invasive lines and drainages, long-term anticoagulation with warfarin with a targeted INR and additional antiplatelet therapy can be started. Recently, a report has been published showing fewer strokes in pediatric EXCOR patients using a triple antiplatelet regimen [64]. 
While the proportion of patients who develop neurological dysfunction after implantation of pulsatile devices has been documented to be approximately $19-30 \%$, the incidence of cerebral strokes in children supported by cf-VADs has not been well explored. A recent report from EUROMACS suggests that it may be as low as 0.1 events per patient year [29]. Similar to the EXCOR, UFH is started postoperatively and then switched to oral anticoagulation. Antiplatelet therapy is in most cases necessary and seems to be meaningful as the pump chamber lays intracorporeal.

\section{Conclusions}

Prolonged durable support in children of all ages and patients with CHD with VADs permits good survival to transplantation. While the Berlin Heart EXCOR remains the "golden standard" for small children, if biventricular is needed, and in some CHD scenarios, an increased miniaturization of VADs has increased cf-device use in these patient. Still, patient and device selection in these patients remain challenging and come with the need for care providers specialized in the field of pediatric/CHD MCS/VAD treatment.

\section{Conflict of interest}

The author does not have any conflict of interest concerning this chapter.

\section{Appendices and nomenclature}

BiVAD

CHD

ECMO

EXCOR

$\mathrm{HF}$

HTx

LVAD

MCS

UFH

TAH

VAD biventricular assist device

congenital heart disease

extracorporeal membrane oxygenation

Berlin Heart pediatric EXCOR

heart failure

heart transplantation

left ventricular assist device

mechanical circulatory support

unfractionated heparin

total artificial heart

ventricular assist device 


\section{Author details}

Martin Schweiger and Michael Huebler

Address all correspondence to: martinl.schweigerr@kispi.uzh.ch

Department of Congenital Cardiovascular Surgery, University Children's Hospital, Zurich, Switzerland

\section{References}

[1] Adachi I, Fraser Jr CD. Mechanical circulatory support for infants and small children. Seminars in Thoracic and Cardiovascular Surgery. Pediatric Cardiac Surgery Annual. 2011;14(1):38-44

[2] Almond CS et al. Waiting list mortality among children listed for heart transplantation in the United States. Circulation. 2009;119(5):717-727

[3] West LJ et al. ABO-incompatible heart transplantation in infants. The New England Journal of Medicine. 2001;344(11):793-800

[4] Schweiger $M$ et al. Pediatric heart transplantation. The Journal of Thoracic Disease. 2015;7(3):552-559

[5] Weiss J, Kocher M, Immer FF. International collaboration and organ exchange in Switzerland. The Journal of Thoracic Disease. 2015;7(3):543-548

[6] Fraser Jr CD et al. Prospective trial of a pediatric ventricular assist device. The New England Journal of Medicine. 2012;367(6):532-541

[7] Dipchand AI et al. The registry of the international society for heart and lung transplantation: Eighteenth official pediatric heart transplantation report-2015; focus theme: Early graft failure. The Journal of Heart and Lung Transplantation. 2015;34(10):1233-1243

[8] Reinhartz O et al. Multicenter experience with the thoratec ventricular assist device in children and adolescents. The Journal of Heart and Lung Transplantation. 2001;20(4):439-448

[9] Blume ED et al. Outcomes of children bridged to heart transplantation with ventricular assist devices: A multi-institutional study. Circulation. 2006;113(19):2313-2319

[10] Krabatsch T et al. Improvements in implantable mechanical circulatory support systems: Literature overview and update. Herz. 2011;36(7):622-629

[11] Feldman D et al. The 2013 international society for heart and lung transplantation guidelines for mechanical circulatory support: Executive summary. The Journal of Heart and Lung Transplantation. 2013;32(2):157-187

[12] Morales DL et al. Bridging children of all sizes to cardiac transplantation: The initial multicenter North American experience with the Berlin Heart EXCOR ventricular assist device. The Journal of Heart and Lung Transplantation. 2011;30(1):1-8 
[13] Kirklin JK et al. The Fourth INTERMACS Annual Report: 4000 implants and counting. The Journal of Heart and Lung Transplantation. 2012;31(2):117-126

[14] Krabatsch $\mathrm{T}$ et al. Mechanical circulatory support-results, developments and trends. Journal of Cardiovascular Translational Research. 2011;4(3):332-339

[15] Blume ED et al. Second annual pediatric interagency registry for mechanical circulatory support (Pedimacs) report: Pre-implant characteristics and outcomes. Journal of Heart and Lung Transplantation. 2017;37(1):38-45

[16] Fan $Y$ et al. Factors associated with the need of biventricular mechanical circulatory support in children with advanced heart failure. European Journal of Cardio-Thoracic Surgery. 2013;43(5):1028-1035

[17] Karl TR, Horton SB, Brizard C. Postoperative support with the centrifugal pump ventricular assist device (VAD). Seminars in Thoracic and Cardiovascular Surgery. Pediatric Cardiac Surgery Annual. 2006;9(1):83-91

[18] Warnecke $H$ et al. Mechanical left ventricular support as a bridge to cardiac transplantation in childhood. European Journal of Cardio-Thoracic Surgery. 1991;5(6):330-333

[19] Hetzer R et al. Mechanical cardiac support in the young with the Berlin Heart EXCOR pulsatile ventricular assist device: 15 years' experience. Seminars in Thoracic and Cardiovascular Surgery. Pediatric Cardiac Surgery Annual. 2006;9:99-108

[20] Konertz W et al. Clinical experience with the MEDOS HIA-VAD system in infants and children: A preliminary report. The Annals of Thoracic Surgery. 1997;63(4):1138-1144

[21] Schweiger $M$ et al. Paediatric ventricular assist devices: Current achievements. Swiss Medical Weekly. 2013;143:w13804

[22] Conway J et al. Delineating survival outcomes in children $<10 \mathrm{~kg}$ bridged to transplant or recovery with the Berlin Heart EXCOR Ventricular Assist Device. JACC Heart Fail. 2015;3(1):70-77

[23] Morales DL et al. Berlin Heart EXCOR use in patients with congenital heart disease. The Journal of Heart and Lung Transplantation. 2017;36(11):1209-1216

[24] Adachi I et al. The miniaturized pediatric continuous-flow device: Preclinical assessment in the chronic sheep model. The Journal of Thoracic and Cardiovascular Surgery. 2017;154(1):291-300

[25] Cabrera AG et al. Outcomes of pediatric patients supported by the HeartMate II left ventricular assist device in the United States. The Journal of Heart and Lung Transplantation. 2013;32(11):1107-1113

[26] Conway J et al. Global experience with the Heartware HVAD ${ }^{\circledR}$ in pediatric patients: A preliminary analysis. JHLT. 2016;35(4S):1

[27] Conway J et al. Now how do we get them home? Outpatient care of pediatric patients on mechanical circulatory support. Pediatric Transplantation. 2016;20(2):194-202 
[28] Schweiger Met al. Outpatient management of intra-corporeal left ventricular assist device system in children: A multi-center experience. American Journal of Transplantation. 2015;15(2):453-460

[29] Schweiger $M$ et al. Cerebral strokes in children on intracorporeal ventricular assist devices: Analysis of the EUROMACS registry. European Journal of Cardio-Thoracic Surgery. 2018;53(2):416-421

[30] Blume ED et al. Outcomes of children implanted with ventricular assist devices in the United States: First analysis of the pediatric interagency registry for mechanical circulatory support (PediMACS). The Journal of Heart and Lung Transplantation. 2016;35(5):578-584

[31] Stiller B et al. Pneumatic pulsatile ventricular assist devices in children under 1 year of age. European Journal of Cardio-Thoracic Surgery. 2005;28(2):234-239

[32] Poh CL et al. Ventricular assist device support in patients with single ventricles: The Melbourne experience. Interactive Cardiovascular and Thoracic Surgery. 2017;25(2):310-316

[33] Adachi I et al. Outpatient management of a child with bidirectional Glenn shunts supported with implantable continuous-flow ventricular assist device. The Journal of Heart and Lung Transplantation. 2016;35(5):688-690

[34] Weinstein $S$ et al. The use of the Berlin Heart EXCOR in patients with functional single ventricle. The Journal of Thoracic and Cardiovascular Surgery. 2014;147(2):697-704 (discussion 704-5)

[35] Irving CA et al. Successful bridge to transplant with the Berlin Heart after cavopulmonary shunt. The Journal of Heart and Lung Transplantation. 2009;28(4):399-401

[36] Adachi I et al. Mechanically assisted Fontan completion: A new approach for the failing Glenn circulation due to isolated ventricular dysfunction. The Journal of Heart and Lung Transplantation. 2016;35(11):1380-1381

[37] Throckmorton AL et al. A viable therapeutic option: Mechanical circulatory support of the failing Fontan physiology. Pediatric Cardiology. 2013;34(6):1357-1365

[38] Giridharan GA et al. Performance evaluation of a pediatric viscous impeller pump for Fontan cavopulmonary assist. The Journal of Thoracic and Cardiovascular Surgery. 2013; 145(1):249-257

[39] Haggerty CM et al. Experimental and numeric investigation of Impella pumps as cavopulmonary assistance for a failing Fontan. The Journal of Thoracic and Cardiovascular Surgery. 2012;144(3):563-569

[40] Pretre R et al. Right-sided univentricular cardiac assistance in a failing Fontan circulation. The Annals of Thoracic Surgery. 2008;86(3):1018-1020

[41] Rodefeld MD et al. Cavopulmonary assist for the univentricular Fontan circulation: Von Karman viscous impeller pump. The Journal of Thoracic and Cardiovascular Surgery. 2010;140(3):529-536 
[42] Pace Napoleone C et al. Ventricular assist device in a failing total cavopulmonary connection: A new step-by-step approach. Interactive Cardiovascular and Thoracic Surgery. 2017;26(2):341-342

[43] Wells D, Villa CR, Simon Morales DL. The 50/50 cc total artificial heart trial: Extending the benefits of the total artificial heart to underserved populations. Seminars in Thoracic and Cardiovascular Surgery. Pediatric Cardiac Surgery Annual. 2017;20:16-19

[44] Steiner JM et al. Durable mechanical circulatory support in teenagers and adults with congenital heart disease: A systematic review. International Journal of Cardiology. 2017; 245:135-140

[45] Stokes MB et al. Successful bridge to Orthotopic cardiac transplantation with implantation of a HeartWare HVAD in management of systemic right ventricular failure in a patient with transposition of the great arteries and previous atrial switch procedure. Heart, Lung \& Circulation. 2016;25(5):e69-e71

[46] VanderPluym CJ et al. Outcomes following implantation of mechanical circulatory support in adults with congenital heart disease: An analysis of the interagency registry for mechanically assisted circulatory support (INTERMACS). Journal of Heart and Lung Transplantation. 2017;37(1):89-99

[47] Tanoue Y, Jinzai Y, Tominaga R. Jarvik 2000 axial-flow ventricular assist device placement to a systemic morphologic right ventricle in congenitally corrected transposition of the great arteries. Journal of Artificial Organs. 2016;19(1):97-99

[48] Maly J et al. Bridge to transplantation with long-term mechanical assist device in adults after the mustard procedure. The Journal of Heart and Lung Transplantation. 2015;34(9):1177-1181

[49] Dakkak AR et al. Implanting a nonpulsatile axial flow left ventricular assist device as a bridge to transplant for systemic ventricular failure after a mustard procedure. Experimental and Clinical Transplantation. 2015;13(5):485-487

[50] Schweiger M et al. Biventricular failure in dextro-transposition of the great arteries corrected with the Mustard procedure: VAD support of the systemic ventricle is enough. The International Journal of Artificial Organs. 2015;38:233-235

[51] Morales DLS et al. Worldwide experience with the Syncardia Total artificial heart in the pediatric population. ASAIO Journal. 2017;63(4):518-519

[52] Glass L et al. Continuous-flow, implantable biventricular assist device as bridge to cardiac transplantation in a small child with restrictive cardiomyopathy. Journal of Heart and Lung Transplantation. 2017;37(1):173-174

[53] Stein ML et al. HeartWare HVAD for biventricular support in children and adolescents: The Stanford experience. ASAIO Journal. 2016;62(5):e46-e51

[54] Schweiger $\mathrm{M}$ et al. Biventricular intracorporeal ventricular assist device in a 10-year-old child. The International Journal of Artificial Organs. 2016;39(1):48-50 
[55] Peng E et al. An extended role of continuous flow device in pediatric mechanical circulatory support. The Annals of Thoracic Surgery. 2016;102(2):620-627

[56] Ovroutski S et al. Two pumps for single ventricle: Mechanical support for establishment of biventricular circulation. The Annals of Thoracic Surgery. 2017;104(2):e143-e145

[57] Fan $\mathrm{Y}$ et al. Outcomes of ventricular assist device support in young patients with small body surface area. European Journal of Cardio-Thoracic Surgery. 2011;39(5):699-704

[58] Reinhartz $\mathrm{O}$ et al. Thoratec ventricular assist devices in pediatric patients: Update on clinical results. ASAIO Journal. 2005;51(5):501-503

[59] Schweiger M et al. Complication profile of the Berlin Heart EXCOR biventricular support in children. Artificial Organs. 2013;37:730-735

[60] Schechter T et al. Unfractionated heparin dosing in young infants: Clinical outcomes in a cohort monitored with anti-factor Xa levels. Journal of Thrombosis and Haemostasis. 2012;10(3):368-374

[61] Guervil DJ et al. Activated partial thromboplastin time versus antifactor Xa heparin assay in monitoring unfractionated heparin by continuous intravenous infusion. The Annals of Pharmacotherapy. 2011;45(7-8):861-868

[62] Schweiger M, Hubler M, Albisetti M. Heparin anticoagulation monitoring in patients supported by ventricular assist devices. ASAIO Journal. 2015;61(5):487-488

[63] Schweiger $M$ et al. Acute chemotherapy-induced cardiomyopathy treated with intracorporeal left ventricular assist device in an 8-year-old child. ASAIO Journal. 2013;59(5): 520-522

[64] Rosenthal DN et al. Impact of a modified anti-thrombotic guideline on stroke in children supported with a pediatric ventricular assist device. The Journal of Heart and Lung Transplantation. 2017;36(11):1250-1257 
